\title{
GRAPHICAL MODELS FOR LOCALIZATION OF THE SEIZURE FOCUS FROM INTERICTAL INTRACRANIAL EEG
}

\author{
Justin Dauwels ${ }^{+}$, Emad Eskandar*, Andy Cole ${ }^{*}$, Dan Hoch* ${ }^{*}$ Rodrigo Zepeda*, Sydney S. Cash* \\ ${ }^{+}$School of Electrical and Electronic Engineering \\ Nanyang Technological University (NTU), Singapore \\ e-mail: justin@dauwels.com \\ ${ }^{*}$ Massachusetts General Hospital and Harvard Medical School, Cambridge, MA, USA
}

\begin{abstract}
Decision algorithms are developed that use periods of intracranial non-seizure (interictal) EEG to localize epileptogenic networks. Depth and surface recordings are considered from 5 and 6 patients respectively. The proposed algorithms combine spectral and multivariate statistics in a decisiontheoretic framework to automatically delineate the seizure onset area. In the case of depth recordings, we apply standard binary classification algorithms, including linear and quadratic discriminative analysis. For the surface recordings, novel decision algorithms are developed, based upon graphical models. The outcomes from the algorithms for both depth and surface recordings are in good agreement with the determination of the seizure focus by clinicians from ictal EEG. In the long term, the proposed approach may lead to shorter hospitalization of intractable-epilepsy patients, since it does not rely on ictal EEG.

\section{INTRODUCTION}

Approximately 50 million people worldwide have epilepsy. More than 50 percent of those suffer from localization-related epilepsy. Unfortunately, $30 \%$ of these patients continue to have seizures despite maximal medical therapy (see, e.g., [1]). Furthermore, many patients suffer from considerable side effects of the medications. On the other hand, regional surgical resection may provide seizure reduction or even cure [2]. However, it is of crucial importance to reliably localize the epileptic brain area(s). At present, one relies mostly on (scalp or intracranial) EEG that contains seizure activity ("ictal EEG" or "seizure" EEG) to determine the seizure onset area; since seizures usually do not occur frequently, recordings must last a long time (from several days to several weeks) until sufficient seizures have occurred (typically between 3 and 5).

In this paper, we investigate whether non-seizure (a.k.a. interictal) intracranial EEG can be used to localize epileptic brain tissue. In particular, we develop novel statistical decision algorithms that detect, combine, and leverage various abnormal interictal electrophysiological characteristics to delineate epileptic brain tissue (see Fig. 1(a)).
\end{abstract}

In order to delineate the seizure onset zone from nonseizure EEG, we will exploit two phenomena: slowing and locally enhanced EEG synchrony ("hypersynchrony"). Signals recorded from damaged cortex often seem to be "slower", i.e., contain more power at low frequencies. Second, a unifying principle emerging from decades of intense research is that seizures are a property of abnormally firing neurons that, entrained by an imbalance of excitation and inhibition, discharge synchronously in a critical ensemble [3]. Moreover, several studies have suggested that multivariate analysis of seizure-free (rest) EEG, in which the relationship between different channels of activity are compared, may help to delineate epileptogenic cortex (e.g., [4, 5]; see also [6] for a recent review).

No study so far, however, has combined several electrophysiological signatures to localize the seizure onset zone from interictal EEG. Virtually all studies focus on one particular signature, and report negative results, showing that one signature does not suffice to delineate seizure onset reliably from interictal EEG (e.g., [4, 5]). This paper demonstrates that a combined approach, founded on algorithmic decision making procedures, substantially improves performance. In future work, we will integrate more signatures into our method (e.g., high-frequency bursts and interictal spikes) to further improve its reliability.

In the long term, one may therefore no longer need to rely on seizure EEG, but instead use short non-seizure EEG recordings to determine the seizure onset area; this would drastically reduce the hospitalization time for intractableepilepsy patients.

This paper is structured as follows. In Section 2 we describe our EEG data and the pre-processing we carried out. In Section 3 we briefly describe our algorithms that merge univariate and multivariate measures to infer the seizure focus, and discuss their performance. At the end of the paper we offer some conclusions.

\section{EEG DATA AND PREPROCESSING}

We investigated data from 11 patients with intractable localized epilepsy who had undergone intracranial investigation 
using intracortical electrodes (depth) and/or subdural surface electrodes (grid). In particular, we analyzed data from 5 patients with only depth electrodes and 6 patients with both grid and depth electrodes. (In the near future, we will investigate data from patients with non-localized epilepsy as well, which will serve as control population.) The depth electrodes ( 5 per hemisphere) contain multiple channels (6 to 8 ); the grids typically contain $8 \times 8$ electrodes. In each case a segment of data 1 hour long and at least 24 hours separated from seizure activity was examined. (In later studies, we will investigate whether smaller separation from seizure activity affects the predictability). The data was band-pass filtered between 1 and $200 \mathrm{~Hz}$, and a notch filter was applied to remove the $60 \mathrm{~Hz}$ power signal components. Before computing the univariate and multivariate measures, each EEG signal was normalized (mean subtracted, divided by standard deviation).

So far, we have applied one univariate measure (relative power), and several multivariate measures, including Pearson correlation coefficient [7], magnitude coherence [7], phase synchrony [8], and omega complexity [9].

For depth electrodes, the univariate measures were applied to all channels of a given depth electrode, and then averaged over all channels, resulting in one average value per depth electrode array. Pairwise synchrony measures (correlation coefficient, magnitude coherence, phase synchrony) were applied to all pairs of channels and then averaged, while omega complexity was calculated from all channels simultaneously. This resulted in a single synchrony value per depth electrode array. The measures varied considerably across patients necessitating a normalization procedure. We divide each measure by the computed average of all depth electrodes of a given patient.

For grid electrodes, the univariate measures were applied to the signal recorded by each electrode; this leads to univariate maps across the grid. For the pairwise synchrony measures, we calculated local synchrony by averaging the pairwise value between the electrode of interest and all of its neighbors. We applied omega complexity simultaneously to the electrode of interest and all its nearest neighbors. Because electrodes at the edge of the grid have fewer neighbors, we normalized the synchrony measures by the number of adjacent electrodes; we divided the measures by the average computed over all electrodes with the same neighborhood size: the 4 corners (neighborhood size 3 ), the other channels at the grid boundaries (size 5), and the inner channels (size 8). From these calculations we obtained local synchrony maps (one for each synchrony measure; normalized and unnormalized), which display the local synchrony of the grid electrodes.

\section{DECISION ALGORITHMS TO DELINEATE SEIZURE ONSET ZONE}

In this section, we elaborate on our decision algorithms for localizing the seizure onset from interictal depth and surface recordings. Since those two types of recordings are quite distinct, we have developed separate algorithms for each type of

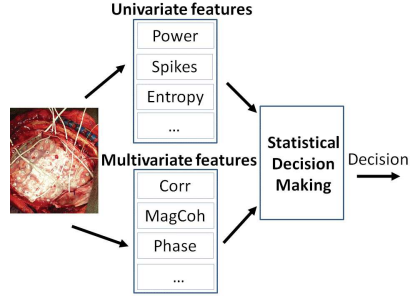

(a)

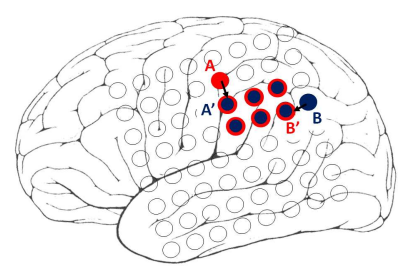

(b)

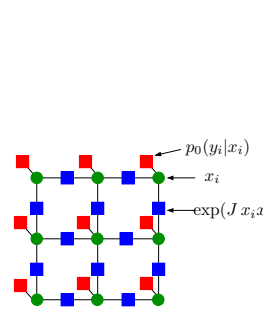

(c)

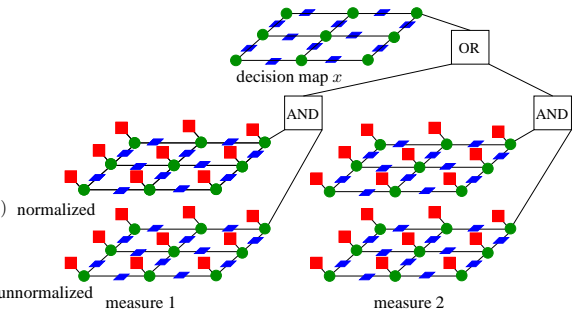

(d)
Fig. 1. Decision making algorithm and graphical representation of measures of accuracy. a) Diagram of the decision making procedure, which combines various measures to compute a probability map of seizure onset; the predicted seizure onset zone is determined as the area in which the seizure onset probability is above a certain threshold. b) Example in which the decision (in red) can be compared to what was determined clinically (in blue). c) Ising model encodes spatial continuity of the measures. d) Logical AND gates merge the normalized and unnormalized values of each measure; logical OR gates merge the different univariate and multivariate measures.

recording. An important difference between depth and grid recordings is the spacing between electrodes: The spacing between depth electrodes is fairly large (several centimeters), and each depth electrode can be treated separately in the decision making; in contrast, the distance between neighboring grid electrodes is much smaller $(5-7 \mathrm{~mm})$, and consequently, the measurements from nearby grid electrodes are often correlated; this spatial correlation needs to be taken into account in the decision making. We first describe our algorithms for the depth recordings, next we elaborate on surface recordings.

\subsection{Depth Recordings}

We aim to determine which depth electrodes are located inside the seizure focus; that problem may be viewed as binary classification, where each electrode is classified separately [10]. We utilize a supervised learning paradigm in which the clinical determinations of seizure focus (gold standard) are used to train binary classifiers with the (normalized) univariate and multivariate measures as input features. Specifically, we conduct linear and quadratic discriminant analysis (LDA and QDA) where we tested all possible combinations of the (normalized) univariate and multivariate measures as features [10]. In all our experiments, we compute the classification rate, specificity, and sensitivity through leave-one-out crossvalidation [10]. 


\subsection{Surface Recordings}

Similarly as for depth recordings, our objective is to determine which grid electrodes are localized inside the seizure onset zone. This problem may also be viewed as binary classification, however, now we need to treat the electrodes jointly. Since the univariate and multivariate measures are prone to noise, they vary sometimes significantly across the grid; those fluctuations are random in nature, and do not necessarily reflect abnormal electrophysiological markers. To overcome this issue, we have developed a novel statistical decision algorithm that exploits the correlation between grid recordings to smoothen the random fluctuations across the grid. The algorithm makes use of the statistical distribution of the univariate and multivariate measures across electrode locations and patients; it computes how likely any given electrode is located inside the seizure focus, given the univariate and multivariate statistics, thereby taking correlations between neighboring electrodes into account. An electrode is considered to be part of the seizure onset zone if the posterior probability of belonging to the seizure onset (computed in the previous step) is above a certain threshold; the latter is computed adaptively, and depends on the posterior probabilities. In the following, we will outline the underlying mathematical formalism.

For each surface electrode, we need to decide whether or not it is located inside the seizure focus. We associate a binary variable $x_{i}$ with each surface electrode $E_{i}$ (with $i=1,2, \ldots, N$, and $N$ is the number of surface electrodes), where $x_{i}=1$ if the electrode $E_{i}$ is located inside the seizure focus, and $x_{i}=-1$ otherwise. Formally, our objective is to infer the binary sequence $x=x_{1}, x_{2}, \ldots, x_{N}$ from the univariate and multivariate statistics. For now, let us consider one measure $y$, e.g., relative power. Similarly as in the depth cases, we normalize that measure, resulting in normalized values $y^{\prime}$. Both the normalized values $y^{\prime}$ and unnormalized values $y$ are taken into account in our decision algorithm. We will first focus on the former. We compute a histogram of the normalized values, across all electrodes and all EEG segments of a given length, denoted by $f_{H}\left(y^{\prime}\right)$; that histogram is an approximation of the (unknown) probability density function $f\left(y^{\prime}\right)$ of the (normalized) measure $y^{\prime}$. From the histogram $f_{H}\left(y^{\prime}\right)$, we compute the cumulative histogram $F_{H}\left(y^{\prime}\right)$, which is an approximation of the probability distribution $F\left(y^{\prime}\right)$ of $y^{\prime}$. From $F_{H}\left(y^{\prime}\right)$ we define the likelihood $p\left(y_{i}^{\prime} \mid x_{i}\right)$ that the electrode at hand is located inside the seizure focus, i.e., $p\left(y_{i}^{\prime} \mid x_{i}=1\right) \propto F_{H}^{\alpha}\left(y_{i}^{\prime}\right)$ and $p\left(y_{i}^{\prime} \mid x_{i}=-1\right) \propto\left(1-F_{H}^{\alpha}\left(y_{i}^{\prime}\right)\right)$, with exponent $\alpha>1$. Since our objective is to infer $x$ for given $y^{\prime}$, only the ratio of $p\left(y_{i}^{\prime} \mid x_{i}=1\right)$ and $p\left(y_{i}^{\prime} \mid x_{i}=-1\right)$ matters, which is equal to the ratio of $F_{H}^{\alpha}\left(y_{i}^{\prime}\right)$ and $\left(1-F_{H}^{\alpha}\left(y_{i}^{\prime}\right)\right)$.

In addition to the information from the univariate and multivariate statistics, we also have prior knowledge about the seizure focus. Usually the seizure focus is located in one area or a few areas, i.e., the seizure focus typically does not consist of numerous disconnected areas. Moreover, several neighboring surface electrodes are typically located in the seizure focus. As a consequence, if an electrode is part of the seizure focus, then probably its neighbors are as well. We encode this continuity constraint with an Ising model:

$$
p(x) \propto \prod_{i, j \in N(i)} \exp \left(J x_{i} x_{j}\right)
$$

where $N(i)$ are the nearest neighbors of electrode $i$ and $J>0$ is a real number. By combining the prior $p(x)$ with the likelihood functions $p\left(y_{i}^{\prime} \mid x_{i}\right)$ we obtain a model $p\left(y^{\prime}, x\right)$ that relates the decisions $x$ to the normalized measure $y^{\prime}$ :

$$
p\left(y^{\prime}, x\right) \propto \prod_{i, j \in N(i)} \exp \left(J x_{i} x_{j}\right) \prod_{i} p\left(y_{i}^{\prime} \mid x_{i}\right) .
$$

The model $p\left(y^{\prime}, x\right)$ is depicted in Fig. 1(c); more specifically, the latter shows a factor graph that represents the factorization (2) of $p\left(y^{\prime}, x\right)$ [11]. The green circles correspond to the variables $x_{i}$, the blue and red squares represent the factors $\exp \left(J x_{i} x_{j}\right)$ and $p\left(y_{i}^{\prime} \mid x_{i}\right)$ respectively. A circle is connected to a square if and only if the variable associated with the circle occurs in the factor associated with the square [11]. In the factorization (2), $x_{i}$ occurs in (two, three, or four) factors $\exp \left(J x_{i} x_{j}\right)$ and one factor $p\left(y_{i}^{\prime} \mid x_{i}\right)$, and consequently each green circle in Fig. 1(c) is connected to (two, three, or four) blue squares and one red square.

If both normalized and unnormalized values are abnormal at electrode $E_{i}$, the latter may be inside the seizure focus, otherwise it is probably not. Similarly to $p\left(y^{\prime}, x\right)(2)$, we introduce model $p(y, x)$ for the unnormalized values $y$. We merge models $p(y, x)$ and $p\left(y^{\prime}, x\right)$ through logical AND gates:

$$
p(\cdot)=p\left(y, x^{\prime \prime}\right) p\left(y^{\prime}, x^{\prime}\right) \prod_{i} \delta\left[\operatorname{AND}\left(x_{i}^{\prime}, x_{i}^{\prime \prime}\right)-x_{i}\right],
$$

where $x^{\prime}$ and $x^{\prime \prime}$ are auxiliary binary sequences, $\operatorname{AND}(z$, $\left.z^{\prime}\right)$ stands for the logical AND of the binary variables $z$ and $z^{\prime}$, and $\delta[$.$] is the Kronecker delta. The auxiliary binary vari-$ ables $x_{i}^{\prime}$ and $x_{i}^{\prime \prime}$ (with $i=1,2, \ldots, N$ ) are associated with the electrodes $E_{i}$; they may be viewed as intermediate binary decision variables that encore whether the normalized and unnormalized values $y^{\prime}$ and $y$ respectively are abnormal, whereas $x_{i}$ encodes whether both $y$ and $y^{\prime}$ are abnormal simultaneously.

So far, we have assumed that only one measure $y$ is available. In practice, we compute several univariate and multivariate measures $\mathbf{y}=y^{(1)}, y^{(2)}, \ldots, y^{(M)}$ (with $M$ the number of measures), and we wish to combine them to delineate the seizure focus. The decisions $x^{(1)}, x^{(2)}, \ldots, x^{(M)}$ are combined together through logical OR gates, resulting in the binary decision sequence $x$ that encodes whether any of the measures is abnormal. The overall statistical model is depicted in Fig. 1(d) for $M=2$ measures; it contains an Ising model (1) for each normalized and unnormalized measure $y^{\prime(m)}$ and $y^{(m)}$ respectively (cf. Fig. 1(c)). The Ising models for the normalized and unnormalized values of a measure are coupled through logical AND gates; the different measures are merged through logical OR gates. In Fig. 1(d) logical OR 
and AND gates are only shown for one set of variables $x_{i}^{(1)}$, $x_{i}^{(2)}$, and $x_{i}$, so as not to overload the figure.

An electrode $E_{i}$ is considered to be located inside the seizure focus (and hence $\hat{x}_{i}=1$ ) if and only if $p\left(x_{i}=\right.$ $\left.1 \mid \mathbf{y}, \mathbf{y}^{\prime}\right)>\rho p_{\max }$, where $p_{\max }=\max _{i} p\left(x_{i}=1 \mid \mathbf{y}, \mathbf{y}^{\prime}\right)$ is the maximum evidence, and $0<\rho<1$. In principle, the marginals $p\left(x_{i}=1 \mid \mathbf{y}, \mathbf{y}^{\prime}\right)$ may be computed by marginalizing the overall model [11]. However, the latter is impractical for the model at hand, since it involves sums over an exponential number of configurations. Instead we compute the marginals $p\left(x_{i}=1 \mid \mathbf{y}, \mathbf{y}^{\prime}\right)$ approximately by applying sum-product message passing to the cyclic graph depicted in Fig. 1(d) [11]. The algorithm may be derived straightforwardly, since all involved variables are binary.

\subsection{Numerical results}

Here we present results from our decision algorithms. Due to space constraints, we limit ourselves to results for the most discriminative pair of features, i.e., relative power and correlation coefficient. Our experiments have shown that adding more features only slightly improves those results. In the depth cases (see Fig. 2(a)), the specificity and sensitivity using relative power alone is $78 \%$ and $88 \%$ respectively; for correlation coefficient it is $29 \%$ and $80 \%$ respectively. When combined the specificity and sensitivity increase to $80 \%$ and $88 \%$ respectively, which is a minor improvement over the results based on relative power alone. Those results were obtained with QDA (through leave-one-out crossvalidation, with EEG segments of 45min), which consistently outperformed LDA for the data at hand.

For the grid cases, we compute distance scores: for each electrode in the predicted onset area, we compute the distance to the closest electrode in the actual onset area, and vice versa, as illustrated in Fig. 1(b). For example, electrode A in Fig. 1(b) is part of the predicted onset area, and its distance to the actual onset area is $\|\mathrm{AA}\|$; likewise, electrode $\mathrm{B}$ is part of the actual seizure onset area, and its distance to the predicted onset area is $\|\mathrm{BB}\|$. The distances $\|\mathrm{AA}\|,\|\mathrm{BB}\|$, etc. are Euclidean distances computed in grid coordinates; for example, a distance of 2 corresponds to twice the distance between neighboring electrodes; the latter is usually about $5 \mathrm{~mm}$. We compute those distances for each electrode in the actual and predicted onset area, and summarize the statistics by two numbers: the median distance for the actual and predicted onset area. As can be seen from Fig. 2(b) and 2(c), the median distances for actual and predicted onset areas are about $2(10 \mathrm{~mm})$ for relative power and the correlation coefficient alone, and are $0.6(3 \mathrm{~mm})$ and $0.4(2 \mathrm{~mm})$ respectively when combined.

\section{CONCLUSIONS}

In the present work, we have presented algorithms that automatically infer the seizure onset zone from interictal recordings, by exploiting various electrophysiological signatures.

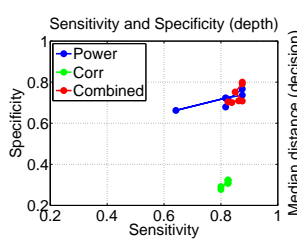

(a)

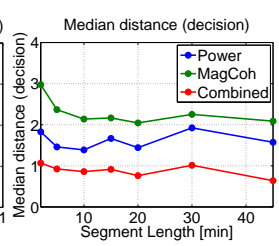

(b)

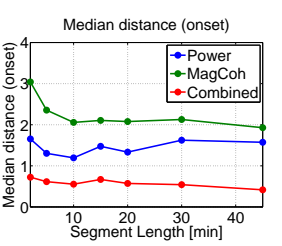

(c)
Fig. 2. Aggregate measures of performance of the decision heuristic based on power, correlation coefficient, or a combination of the two, for EEG segments of varying length (2$45 \mathrm{~min}$ ). a) Sensitivity and specificity for depth cases. b) Median distance between predicted onset and closest actual onset for grid cases. c) Median distance between actual onset and closest predicted onset for grid cases.

With those algorithms in hand, we will in the near future organize a multi-center trial in which short-length interictal data is used to determine the seizure onset zone and a recommended resection is offered prospectively. Outcomes in terms of both seizure freedom and complications will be analyzed.

We will also try to incorporate short-term intra-operative recordings into our algorithm. Demonstration that an accurate seizure onset zone prediction could be obtained from OR recordings only would eventually obviate the need for semichronic invasive recordings.

\section{REFERENCES}

[1] T. Keranen, M. Sillanpaa, et al., "Distribution of seizure types in an epileptic population," Epilepsia 29(1):1-7.

[2] J. Engel Jr., S. Wiebe, et al., "Practice parameter: Temporal lobe and localized neocortical resections for epilepsy, Neurology 60(4): 538-47, 2003.

[3] A. Wyler and A. Ward, Epilepsy: A Window to Brain Mechanism, New York, Raven, 1992.

[4] C. Schevon, J. Cappell, et al., "Cortical abnormalities in epilepsy revealed by local EEG synchrony," Neuroimage 35(1):140-8, 2007.

[5] G. Ortega, L. M. de la Prida, et al., "Synchronization clusters of interictal activity in the lateral temporal cortex of epileptic patients: Intraoperative electrocorticographic analysis," Epilepsia 49(2):269-280, 2008.

[6] Lehnertz K., Bialonski S., et al.,"Synchronization phenomena in human epileptic brain networks," Journal of Neuroscience Methods 183(1):42-48, 2009.

[7] P. Nunez and R. Srinivasan, Electric Fields of the Brain: The Neurophysics of EEG, Oxford University Press, 2006.

[8] J.-P. Lachaux, E. Rodriguez, et al., "Measuring Phase Synchrony in Brain Signals," Human Brain Mapping 8:194-208 (1999).

[9] Saito, N., T. Kuginuki, et al., "Global, regional, and local measures of complexity of multichannel EEG in acute, neurolepticnaive, first-break schizophrenics," Society of Biological Psychiatry 43: 794-802, 1998.

[10] R.O. Duda, P.E. Hart, et al., Pattern Classification (2nd Edition), Wiley-Interscience, October 2000.

[11] Loeliger H.-A., Dauwels J., et al., 2007. The factor graph approach to model-based signal processing. Proceedings of the IEEE 95(6), 1295-1322. 